\title{
Trajectories and characteristics of functional impairment before and after suicide attempt in young adults - a nationwide register-based cohort study
}

\author{
Mo Wang ${ }^{1 *}$ (D, Magnus Helgesson ${ }^{1}$, Syed Rahman ${ }^{1}$, Thomas Niederkrotenthaler ${ }^{2}$ and Ellenor Mittendorfer-Rutz ${ }^{1}$
}

\begin{abstract}
Background: Despite high rates of youth suicide attempt, little is known about patterns of functional impairment in terms of sickness absence and disability pension (SA/DP) before and after an attempt. The aim was to identify SA/DP trajectories among young adults with or without suicide attempt and to describe associations of socio-demographic and clinical factors with such trajectories.

Methods: This is a population-based cohort study of 5385 individuals aged 25-40 years with a first suicide attempt during 2007-2009. One control for each case without suicide attempt was matched by socio-demographic factors. Trajectories of annual SA/DP months over an eight-year period were analysed by group-based trajectory modelling. Associations between socio-demographic and clinical factors with trajectory groups were estimated by chi ${ }^{2}$-test and multinomial logistic regression.

Results: Two groups of suicide attempters had low SA/DP levels over time (62\%). One group had constantly high SA DP levels (16\%). The remaining two groups had increased SA/DP initially, which then decreased at different time points. Socio-demographic and clinical factors were associated with different trajectories $\left(R^{2}=0.44\right)$. Suicide attempters with low levels of SAVDP were likely to be unemployed whereas a larger proportion of those with high levels of SA/DP had psychiatric health care before the suicide attempt, particularly due to schizophrenia and non-affective psychoses or personality disorders.
\end{abstract}

Conclusions: Young suicide attempters even with no/low levels of SA/DP were likely to be marginalised at the labour market. Schizophrenia/non-affective psychoses and personality disorders were important clinical factors for differentiating the levels of SA/DP among young suicide attempters.

Keywords: Sick leave, Disability pension, Suicide attempt, Trajectory

\section{Background}

Suicide attempt is a major and growing public health problem worldwide [1], especially among adolescents and young adults $[2,3]$. Young people with suicide attempt are likely to repeat this behaviour and have an increased risk for subsequent mortality due to suicide [2]. Moreover, the long-term risk of functional impairment is increased in young suicide attempters, particularly impairment related

\footnotetext{
* Correspondence: mo.wang@ki.se

${ }^{1}$ Division of Insurance Medicine, Department of Clinical Neuroscience,

Karolinska Institutet, SE 17177 Stockholm, Sweden

Full list of author information is available at the end of the article
}

to work incapacity, i.e. sickness absence and disability pension (SA/DP) [4]. One recent study has reported that sex, country of birth, schizophrenia, and personality disorders as well as the method of suicide attempt were associated with the subsequent risk of SA and DP in young suicide attempters [5].

Despite the evidence related to the interlinked relationship between suicide attempt and SA/DP, only a few studies have investigated the association between SA/DP and suicide attempt in the general population and in individuals with depressive disorders [6-9]. A major limitation of these studies is that they did not take the course (patterns) of SA/ 
DP into account. When studying such associations, it needs to be considered that suicide attempters comprise a heterogeneous group in terms of aetiology, intent, sociodemographic characteristics, clinical factors such as severity of underlying mental and somatic diseases, psychosocial factors, as well as healthcare seeking behaviour [2]. Moreover, method of suicide attempt is heterogeneous, including acts such as hanging, poisoning, overdosing, or cutting [2]. SA/ DP may also fluctuate over time. Trajectory analysis offers the opportunity to elucidate this heterogeneity and the differential development of time-dependent phenomena [10]. In addition, a comparison with individuals without suicide attempt can provide important insights in peculiarities of $\mathrm{SA} / \mathrm{DP}$ trajectories in young suicide attempters.

\section{Aims}

The aim of this study was to identify trajectories of SA/DP using an observation window of 8 years before and after suicide attempt in young adults and elucidate the relation of socio-demographic and clinical characteristics (i.e. underlying diagnosis-specific mental and somatic inpatient and specialised outpatient care, psychiatric medication, and method of suicide attempt) with such trajectories. Another aim was to compare the trajectories with a group of young individuals without suicide attempt.

\section{Methods and materials}

\section{Study population}

This study base included all individuals resident in Sweden and between 25 and 40 years of age, when treated in inpatient care due to a first suicide attempt during 2007-2009 $(N=6844)$. To assure eligibility for SA/DP particularly before suicide attempt, the age range of young suicide attempters was defined between 25 and 40 years. Individuals with previous suicide attempts from inpatient care 1987-2006 were excluded ( $n=1396,20.4 \%)$. Moreover, individuals with missing information on sociodemographic factors $(n=63,0.9 \%)$ were excluded. The final study population consisted of 5385 individuals with first-time suicide attempt. Suicide attempt was defined based on the International Classification of Diseases (ICD) 10 codes: X60-84 and Y10-Y34. The ICD-10 codes Y10-Y34 (undetermined intent) were included in order to limit underreporting of suicide attempt and to limit temporal and geographic differences in ascertainment procedures $[4,11,12]$. A sensitivity analysis comparing estimates with and without suicide attempt due to undetermined intent yielded similar results.

Individuals between 25 and 40 years of age during 2007 and 2009 and resident in Sweden who did not have any inpatient care due to suicide attempt during 1987-2013 were used as a comparison group $(N=5385)$. For each suicide attempt case, one control was randomly selected and matched on sex, age, education, country of birth, and family situation (see categorisation in Table 1). Matching factors were measured on 31st December of the year preceding baseline (i.e. 2007-2009). The cohort entry date of the control was the date of suicide attempt of the matched case.

\section{Registers}

Individual information was linked through registers by using the unique personal identity number. The following national registers from Swedish authorities were available for each individual retrospectively and prospectively up to 31st December 2013:

1) Longitudinal integration database for health insurance and labour market studies (LISA) from Statistics Sweden: age, sex, country of birth, education, family situation, type of living area, year of emigration, number of days with unemployment, sickness absence, and disability pension.

2) (i) date and diagnoses of in- and specialised outpatient care from the National patient register; (ii) date of death from the Cause of death register; (iii) date, defined daily dose (DDD), and Anatomical Therapeutic Chemical (ATC) Classification System code from the Drug register - all from the National Board of Health and Welfare.

\section{The social insurance system in Sweden}

In Sweden, the Social Insurance Agency grants sickness benefits for all people from the age of 16 who have an income from work or unemployment benefits, and a reduced work capacity due to a disease or injury [13]. Employed individuals have one qualifying day without benefits and selfemployed individuals may have more qualifying days according to the insurance they have chosen. Employees receive sick pay from their employers during the first 14 days. Afterwards, they receive sickness benefits from the Social Insurance Agency. After 7 days of self-certification, a physician certificate is required. DP is granted to people who have a permanently impaired work capacity due to a disease or injury. Since 2003, individuals aged 19-29 years can receive temporary DP.

\section{Sickness absence and disability pension}

The SA and DP days were combined and the mean annual number of SA/DP days from 4 years before to 4 years after baseline was calculated. As SA and DP can be granted for part time, the number of net days was used, e.g., 2 days of half-time sickness benefits equals one net day. Then, the number of annual net days was transformed to number of annual months with SA/DP.

\section{Socio-demographic and clinical factors}

Characteristics of individuals included socio-demographics (i.e., sex, age, education level, country of birth, type of living 
Table 1 Descriptive statistics for all women $(n=2878)$ and men $(n=2507)$ with a first suicide attempt treated in inpatient care 2007-2009 in Sweden ( $N=5385)$

\begin{tabular}{|c|c|c|c|c|c|c|}
\hline \multirow[t]{3}{*}{ Characteristics } & \multicolumn{2}{|l|}{ All } & \multicolumn{2}{|c|}{ Women } & \multicolumn{2}{|l|}{ Men } \\
\hline & $\mathrm{n}$ & $\%$ & $\mathrm{n}$ & $\%$ & $n$ & $\%$ \\
\hline & 5385 & 100 & 2878 & 53.4 & 2507 & 46.6 \\
\hline \multicolumn{7}{|l|}{ Socio-demographic characteristics $^{a}$} \\
\hline \multicolumn{7}{|l|}{ Age } \\
\hline $24-29$ & 2329 & 43.2 & 1242 & 43.2 & 1087 & 43.4 \\
\hline $30-34$ & 1486 & 27.6 & 792 & 27.5 & 694 & 27.7 \\
\hline $35-40$ & 1570 & 29.2 & 844 & 29.3 & 726 & 29.0 \\
\hline \multicolumn{7}{|l|}{ Education (years) ${ }^{*}$} \\
\hline Compulsory $(\leq 9)$ & 1730 & 32.1 & 814 & 28.3 & 916 & 36.5 \\
\hline High school (10-12) & 2716 & 50.4 & 1437 & 49.9 & 1279 & 51.0 \\
\hline University (>12) & 939 & 17.4 & 627 & 21.8 & 312 & 12.4 \\
\hline \multicolumn{7}{|l|}{ Country of birth ${ }^{*}$} \\
\hline Sweden & 4301 & 79.9 & 2227 & 77.4 & 2074 & 82.7 \\
\hline European countries without Sweden ${ }^{b}$ & 218 & 4.0 & 117 & 4.1 & 101 & 4.0 \\
\hline Non-European countries & 866 & 16.1 & 534 & 18.6 & 332 & 13.2 \\
\hline \multicolumn{7}{|l|}{ Type of living area ${ }^{c}$} \\
\hline Big cities & 2050 & 38.1 & 1102 & 38.3 & 948 & 37.8 \\
\hline Medium sized cities & 2015 & 37.4 & 1078 & 37.5 & 937 & 37.4 \\
\hline Small towns/villages & 1320 & 24.5 & 698 & 24.3 & 622 & 24.8 \\
\hline \multicolumn{7}{|l|}{ Family situation $^{*}$} \\
\hline Married ${ }^{d}$ living without children & 163 & 3.0 & 96 & 3.3 & 67 & 2.7 \\
\hline Married ${ }^{d}$ living with children & 1158 & 21.5 & 742 & 25.8 & 416 & 16.6 \\
\hline Single $e^{e}$ living without children & 3340 & 62.0 & 1368 & 47.5 & 1972 & 78.7 \\
\hline Single ${ }^{e}$ living with children & 724 & 13.4 & 672 & 23.3 & 52 & 2.1 \\
\hline \multicolumn{7}{|l|}{ Unemployment ${ }^{*}$} \\
\hline No & 3969 & 73.7 & 2188 & 76.0 & 1781 & 71.0 \\
\hline $1-180$ days & 1122 & 20.8 & 560 & 19.5 & 562 & 22.4 \\
\hline$>180$ days & 294 & 5.5 & 130 & 4.5 & 164 & 6.5 \\
\hline \multicolumn{7}{|l|}{ Clinical characteristics } \\
\hline \multicolumn{7}{|l|}{ Somatic inpatient care ${ }^{*}$, g } \\
\hline No & 2820 & 52.4 & 1311 & 45.6 & 1509 & 60.2 \\
\hline Yes & 2565 & 47.6 & 1567 & 54.4 & 998 & 39.8 \\
\hline \multicolumn{7}{|l|}{ Specialised somatic outpatient care ${ }^{*}$, g } \\
\hline No & 1357 & 25.2 & 641 & 22.3 & 716 & 28.6 \\
\hline Yes & 4028 & 74.8 & 2237 & 77.7 & 1791 & 71.4 \\
\hline \multicolumn{7}{|c|}{ Mental diagnosis from in- or specialised outpatient care ${ }^{*}$, g } \\
\hline No & 2369 & 44.0 & 1275 & 44.3 & 1094 & 43.6 \\
\hline Schizophrenia non-affective psychoses & 152 & 2.8 & 51 & 1.8 & 93 & 3.7 \\
\hline Affective disorders & 801 & 14.9 & 496 & 17.2 & 256 & 10.2 \\
\hline Substance abuse & 913 & 17.0 & 258 & 9.0 & 605 & 24.1 \\
\hline Neurotic, somatoform and stress-related disorders & 733 & 13.6 & 473 & 16.4 & 234 & 9.3 \\
\hline Personality disorders & 213 & 4.0 & 119 & 4.1 & 49 & 2.0 \\
\hline Other mental disorders & 204 & 3.8 & 103 & 3.6 & 94 & 3.7 \\
\hline
\end{tabular}


Table 1 Descriptive statistics for all women $(n=2878)$ and men $(n=2507)$ with a first suicide attempt treated in inpatient care 2007-2009 in Sweden ( $N=5385)$ (Continued)

\begin{tabular}{|c|c|c|c|c|c|c|}
\hline \multirow[t]{3}{*}{ Characteristics } & \multicolumn{2}{|l|}{ All } & \multicolumn{2}{|c|}{ Women } & \multicolumn{2}{|l|}{ Men } \\
\hline & $\mathrm{n}$ & $\%$ & $\mathrm{n}$ & $\%$ & $n$ & $\%$ \\
\hline & 5385 & 100 & 2878 & 53.4 & 2507 & 46.6 \\
\hline \multicolumn{7}{|l|}{ Antidepressants $^{*} h, i$} \\
\hline No antidepressants & 2893 & 53.7 & 1331 & 46.2 & 1562 & 62.3 \\
\hline Low doses & 732 & 13.6 & 434 & 15.1 & 298 & 11.9 \\
\hline Moderate doses & 1020 & 18.9 & 641 & 22.3 & 379 & 15.1 \\
\hline High doses & 740 & 13.7 & 472 & 16.4 & 268 & 10.7 \\
\hline \multicolumn{7}{|l|}{ Anxiolytics ${ }^{*}$ h, i } \\
\hline No Anxiolytics & 3650 & 67.8 & 1850 & 64.3 & 1800 & 71.8 \\
\hline Low doses & 1288 & 23.9 & 808 & 28.1 & 480 & 19.1 \\
\hline Moderate doses & 267 & 5.0 & 142 & 4.9 & 125 & 5.0 \\
\hline High doses & 180 & 3.3 & 78 & 2.7 & 102 & 4.1 \\
\hline \multicolumn{7}{|l|}{ Sedatives $^{*} \mathrm{~h}, \mathrm{i}$} \\
\hline No sedatives & 3364 & 62.5 & 1695 & 58.9 & 1669 & 66.6 \\
\hline Low doses & 1080 & 20.1 & 631 & 21.9 & 449 & 17.9 \\
\hline Moderate doses & 555 & 10.3 & 322 & 11.2 & 233 & 9.3 \\
\hline High doses & 386 & 7.2 & 230 & 8.0 & 156 & 6.2 \\
\hline \multicolumn{7}{|l|}{ Method of suicide attempt ${ }^{*} \mathrm{~g}, \mathrm{j}$} \\
\hline Violent suicide attempt & 671 & 12.5 & 221 & 7.7 & 450 & 17.9 \\
\hline Non-violent suicide attempt & 4714 & 87.5 & 2657 & 92.3 & 2057 & 82.1 \\
\hline
\end{tabular}

* Significant sex differences

a Measured on 31-Dec of the year preceding suicide attempt

${ }^{b}$ Including other Northern European countries than Sweden and the rest of EU25

c Type of living area: big cities (Stockholm, Gothenburg and Malmö); medium sized cities (cities with more than 90,000 inhabitants within 30 km distance from the centre of the city); small cities/villages/rural

${ }^{\mathrm{d}}$ Married includes all living with partner; cohabiting

e Single comprises divorced, separated, and widowed

${ }^{f}$ Measured four years before suicide attempt

g See methods section for ICD-10 codes

${ }^{h}$ Measured during the year preceding the suicide attempt

i See methods section for ATC codes

${ }^{\mathrm{j}}$ Measured at index suicide attempt

area, family situation, and number of days with unemployment), and clinical characteristics (i.e. diagnosis-specific mental and somatic inpatient and specialised outpatient care, psychiatric medication, and method of suicide attempt) (See Table 1).

Socio-demographics were measured on 31st December of the year preceding suicide attempt and categorised as shown in Table 1. The main diagnosis from previous in- and specialised outpatient care due to somatic disorders was measured during 4 years before suicide attempt up to the year preceding suicide attempt. The individuals with and without at least one previous somatic in- or outpatient care were coded positive and negative for this variable, respectively. The chronologically latest main mental diagnoses from inor specialised outpatient care were categorised according to ICD-10 codes: Schizophrenia and non-affective psychoses (F20-F29); affective disorders (F30-39); substance abuse disorders (F10-F19); neurotic, stress-related and somatoform disorders (F40-F48); personality disorders (F60) and all remaining mental diagnoses (F00-F09, F50-59, F61-F69, F70-F79, F80-F89, F90-F99). Prescription of psychiatric medication, i.e. antidepressants, anxiolytics and sedatives was included and coded according to the ATC codes, namely N06A, N05B and N05C, respectively. Prescribed psychiatric medication was assessed according to DDDs during the year preceding suicide attempt and categorised into 4 groups in relation to DDDs [14]. Psychiatric medication less than 0.5 DDD was grouped as low doses; $0.5-1.5$ DDD as moderate doses while high doses referred to more than 1.5 DDD [15].

Method of the index suicide attempt was defined as violent and non-violent suicide attempts [16] (Table 1). Violent suicide attempts included hanging, use of firearms or knives, jumping from height or in front of vehicles and drowning (ICD-10: X70-X82 and Y20-Y32). Non-violent attempted suicide comprised poisoning: X60-X69 and 
Y10-Y19 in ICD-10. There were 183 (3.4\%) individuals with unspecific events/means (ICD-10: X83-X84 and Y33-Y34) who were grouped into violent suicide attempts according to the description of ICD-10 [17]. A sensitivity analysis categorising the unspecific methods into non-violent suicide attempt did not change the overall results.

\section{Statistical methods}

Firstly, sex differences were measured in the sociodemographic and clinical characteristics among young suicide attempters by a $\mathrm{Chi}^{2}$ test. Then, group-based trajectory modelling was used to measure trajectories of SA/DP among individuals with or without suicide attempt from 4 years before baseline until 4 years after [18]. An annual time-scale was applied in the study, where T-4 represents 4 years before baseline and T4 equals 4 years after baseline. The method is developed in order to identify subgroups of individuals with distinct trajectories during the time of observation. Also, this method could provide the possibility to assess variation and stability over time for each identified subgroup and the proportion of individuals in each group. We tested 2-7 groups until the model of best fit was obtained, as determined by comparing the Bayesian information criterion (BIC). A model with five groups for suicide attempt cohort and a model with three groups for the comparison cohort were found as the best fitting model. The highest estimated probability was used to decide each individual's group belonging. Côté et al. recommended an average probability of $\geq 0.70$ for individuals of a trajectory group [19]. These probabilities for individuals in the suicide attempt cohort and the comparison cohort were 0.91 and 0.96 , respectively, indicating a good fit.

Distributions of socio-demographic and clinical factors in each SA/DP trajectory group among young suicide attempters were explored by $\mathrm{Chi}^{2}$-test and multinomial logistic regression. The likelihood ratio test was applied to evaluate the associations between socio-demographic and clinical factors with type of trajectory group in the full model. Moreover, the strength of these associations was estimated by Nagelkerke pseudo $R^{2}$. Each factor was consecutively excluded and re-included from the full model in order to calculate the differences in $R^{2}$. These differences in $R^{2}$ were used as indicators of the contribution of a given factor to the full model. Additionally, the data on SA/DP for an individual who died or emigrated was considered as missing for the whole year of the event and onwards.

Data processing was performed using the statistical software SAS for Windows version 9.4 (SAS-based procedure "Traj") [18] and SPSS for Windows version 22.0 ( $\mathrm{chi}^{2}$-test and multinomial logistic regression).

\section{Results}

In Table 1 descriptive statistics of the young suicide attempters with regard to characteristics on sociodemographics and clinical factors is shown for all attempters as well as stratified by sex. Of the 5385 individuals who were treated in inpatient care due to a first suicide attempt during 2007-2009, 2878 (53.4\%) were women. In this group, larger proportions of the cohort were of younger age (24-29 years, 43.2\%), had achieved an education at high school level (50.4\%), were born in Sweden (79.9\%), were single and living without children $(62.0 \%)$, and were not registered as unemployed (73.7\%). Men were more often single living without children than women $(78.7 \%$ vs. $47.5 \%)$ while more women than men were single living with children ( $23.3 \%$ vs. $2.1 \%)$. Regarding clinical characteristics, the majority of suicide attempters had specialised outpatient care visits due to somatic disorders $(74.8 \%)$ and used non-violent suicide attempt methods (87.5\%). Substance abuse (17.0\%), affective disorders (14.9\%), and stress-related disorders (13.6\%) were more common than other mental disorders in young adults with suicide attempt. More women than men used psychiatric medication. Additionally, more women than men had previous somatic inpatient care $(54.4 \%$ vs. $39.8 \%)$ while more men had a diagnosis of substance abuse $(24.1 \%$ vs. 9.0\%) and used violent methods of suicide attempt (17.9\% vs. $7.7 \%$ ) than women (Table 1).

\section{Trajectory analyses}

Five different trajectory groups of SA/DP months for the suicide attempt cohort were identified (Fig. 1). The five groups were named as "Lowest constant", "Intermediate constant", "High decreasing", "Low increasing", and "High constant".

Among individuals with suicide attempt, approximately one third (32.1\%) belonged to the "Lowest constant" group. They constantly had no or quite few months of SA/DP over the years before and after suicide attempt. There were $30.0 \%$ of the individuals in the "Intermediate constant" group with an average of 1 month of SA/DP/year during the study period. The "High constant" group included $15.9 \%$ of the individuals. On average, they had around 10 months of SA/DP annually. The "High decreasing" group included $7.5 \%$ of the individuals. Four years before attempt, this group had approximately 4 SA/DP months per year, increasing up to 8 months at T-2, when the level of SA/DP months decreased. The "Low increasing" group included 14.5\% of individuals. They had moderately low level of SA/DP months at T-4 and showed an increasing trend reaching around 8 months at T3 (Fig. 1).

The comparison cohort showed three trajectory groups over time. The three groups were named as "Lowest constant", "Intermediate constant", and "High 

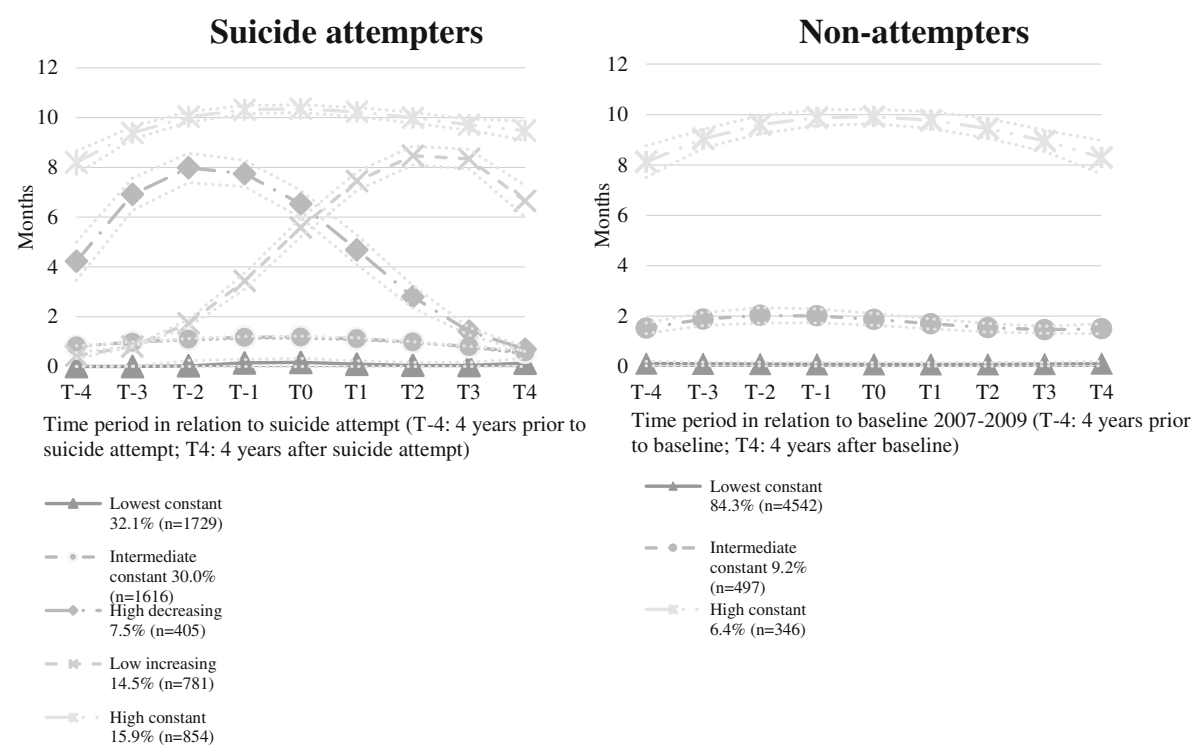

Fig. 1 Trajectory groups of sickness absence and disability pension (SA/DP) months and percentages of individuals with suicide attempt treated from inpatient care in 2007-09 ( $N=5385$ ) and individuals who were living in Sweden during 2007-2009 without suicide attempt ( $N=5385)$ within each trajectory group. The dotted lines represent $95 \%$ confidence intervals

constant". The absolute majority belonged to the "Lowest constant" group (84.3\%). The "Intermediate constant" group included $9.2 \%$ of individuals which is fewer than the suicide attempt group. There were only $6.4 \%$ of the individuals included in the "High constant" group (Fig. 1).

Table 2 shows distributions and associations of sociodemographic and clinical characteristics in each trajectory group among suicide attempters. All socio-demographic and clinical factors were significantly associated with the trajectory groups $(P<0.001)$ in the unadjusted analyses. After mutual adjustment in the full model, the significant results remained except for "method of suicide attempt". Nagelkerke pseudo $R^{2}$ for the full model is 0.436 which indicated an association between socio-demographic and clinical factors with the trajectory groups. The differences in $R^{2}$ indicated that the different factors did not independently have strong effects in the full model, except for mental diagnoses before suicide attempt (Diff. in $R^{2}=0.052$ ) and days of unemployment (Diff. in $R^{2}=0.029$ ), which had a more important role than other factors in the full model.

Individuals in the "Lowest constant" group tended to be men, better educated, younger (24-29 years), born in other countries than Sweden, living in big cities, and unemployed while fewer individuals in this group were single living with children. The "Intermediate constant" group also had more unemployed individuals. In contrast, the "High constant" group included a larger proportion of older individuals living in small towns/villages with lower levels of education, but few individuals were unemployed. Other socio-demographic factors were relatively equally distributed among all the groups.
Regarding the clinical characteristics, more attempters in the "High constant" group had previous in- and specialised outpatient somatic care, a diagnosis of schizophrenia and non-affective psychoses or a personality disorder and use of antidepressants, anxiolytics, and sedatives, particularly high doses of use. The "High decreasing" group also included more individuals with somatic disorders, and low/moderate doses of psychiatric medication. In the "Low increasing" group, a larger proportion of individuals had affective disorders and low doses of psychiatric medication. On the contrary, the "Lowest constant" group showed less specialised somatic and mental care and less use of psychiatric medication compared to other groups (Table 2).

\section{Discussion \\ Main findings}

In this study, five different trajectories of SA/DP were identified over an 8-year period among all 5385 young individuals with a first suicide attempt treated in inpatient care during 2007-2009 in Sweden. More than two thirds of the individuals had no or few annual SA/ DP months before and after suicide attempt. In contrast, $15.9 \%$ of the individuals had constantly high SA/DP months over time. The remaining two groups, including $7.5 \%$ and $14.5 \%$ in each, showed increasing SA/DP firstly and then their annual SA/DP months decreased at different time points. A comparison group of 5385 young individuals without suicide attempt showed three trajectory groups of SA/DP. The absolute majority of these individuals in group had no or very low levels of SA/DP months throughout the study period (93.5\%). All 
Table 2 Distributions and associations of socio-demographic and clinical characteristics in each trajectory group of annual months of sickness absence and disability pension (SA/DP) in individuals with suicide attempt treated in inpatient care in 2007-2009 in Sweden $(N=5385)$

\begin{tabular}{|c|c|c|c|c|c|c|c|c|}
\hline \multirow[t]{3}{*}{ Characteristics } & $\begin{array}{l}\text { Lowest } \\
\text { constant }\end{array}$ & $\begin{array}{l}\text { Intermediate } \\
\text { constant }\end{array}$ & $\begin{array}{l}\text { High } \\
\text { decreasing }\end{array}$ & $\begin{array}{l}\text { Low } \\
\text { increasing }\end{array}$ & $\begin{array}{l}\text { High } \\
\text { constant }\end{array}$ & \multirow[t]{3}{*}{$\begin{array}{l}\text { Pearson's Chi-Square } \\
(p \text {-value })^{k}\end{array}$} & \multirow[t]{3}{*}{$\begin{array}{l}\text { Log-likelihood test } \\
\text { Chi-Square } \\
(p \text {-value) })^{\prime}\end{array}$} & \multirow[t]{3}{*}{$\begin{array}{l}\text { Diff. in } \\
\mathrm{R}^{2 *}\end{array}$} \\
\hline & n (\%) & n (\%) & n (\%) & n (\%) & n (\%) & & & \\
\hline & $1729(32.1)$ & $1616(30.0)$ & $405(7.5)$ & $781(14.5)$ & $854(15.9)$ & & & \\
\hline \multicolumn{9}{|l|}{ Socio-demographic characteristics $^{a}$} \\
\hline \multicolumn{9}{|l|}{ Sex } \\
\hline Men & $1014(58.6)$ & $708(43.8)$ & $148(36.5)$ & $294(37.6)$ & $343(40.2)$ & \multirow[t]{2}{*}{$161.7(<0.001)$} & \multirow[t]{2}{*}{$56.0(<0.001)$} & \multirow[t]{2}{*}{0.007} \\
\hline Women & $715(41.4)$ & $908(56.2)$ & $257(63.5)$ & $487(62.4)$ & $511(59.8)$ & & & \\
\hline \multicolumn{9}{|l|}{ Age } \\
\hline $24-29$ & $895(51.8)$ & $681(42.1)$ & $149(36.8)$ & $355(45.5)$ & $249(29.2)$ & \multirow[t]{3}{*}{$171.4(<0.001)$} & \multirow[t]{3}{*}{$125.4(<0.001)$} & \multirow[t]{3}{*}{0.015} \\
\hline $30-34$ & $447(25.9)$ & $483(29.9)$ & $115(28.4)$ & $207(26.5)$ & $234(27.4)$ & & & \\
\hline $35-40$ & $387(22.4)$ & $452(28.0)$ & $141(34.8)$ & $219(28.0)$ & $371(43.4)$ & & & \\
\hline \multicolumn{9}{|l|}{ Education (years) } \\
\hline Compulsory ( $\leq 9)$ & $667(38.6)$ & $389(24.1)$ & $132(32.6)$ & $208(26.6)$ & $334(39.1)$ & \multirow[t]{3}{*}{$154.1(<0.001)$} & \multirow[t]{3}{*}{$157.9(<0.001)$} & \multirow[t]{3}{*}{0.019} \\
\hline High school (10-12) & $730(42.2)$ & $930(57.5)$ & $218(53.8)$ & $407(52.1)$ & $431(50.5)$ & & & \\
\hline University (>12) & $332(19.2)$ & $297(18.4)$ & 55 (13.6) & $166(21.3)$ & $89(10.4)$ & & & \\
\hline \multicolumn{9}{|l|}{ Country of birth } \\
\hline Sweden & $1164(67.3)$ & $1377(85.2)$ & $354(87.4)$ & $655(83.9)$ & $751(87.9)$ & \multirow[t]{3}{*}{$259.3(<0.001)$} & $135.0(<0.001)$ & \multirow[t]{3}{*}{0.016} \\
\hline $\begin{array}{l}\text { European countries } \\
\text { without Sweden }^{b}\end{array}$ & $103(6.0)$ & $51(3.2)$ & $10(2.5)$ & $25(3.2)$ & $29(3.4)$ & & & \\
\hline Non-European countries & $462(26.7)$ & $188(11.6)$ & $41(10.1)$ & $101(12.9)$ & $74(8.7)$ & & & \\
\hline \multicolumn{9}{|l|}{ Type of living area ${ }^{c}$} \\
\hline Big cities & $736(42.6)$ & $595(36.8)$ & $140(34.6)$ & $295(37.8)$ & $284(33.3)$ & \multirow[t]{3}{*}{$38.6(<0.001)$} & $22.2(<0.05)$ & \multirow[t]{3}{*}{0.003} \\
\hline Medium sized cities & $636(36.8)$ & $593(36.7)$ & $161(39.8)$ & $303(38.8)$ & $322(37.7)$ & & & \\
\hline Small towns/villages & $357(20.6)$ & $428(26.5)$ & $104(25.7)$ & $183(23.4)$ & $248(29.0)$ & & & \\
\hline \multicolumn{9}{|l|}{ Family situation } \\
\hline Married ${ }^{d}$ living without children & $74(4.3)$ & $44(2.7)$ & $11(2.7)$ & $19(2.4)$ & $15(1.8)$ & $128.4(<0.001)$ & $56.0(<0.001)$ & 0.007 \\
\hline Married ${ }^{d}$ living with children & $352(20.4)$ & $419(25.9)$ & $92(22.7)$ & $171(21.9)$ & $124(14.5)$ & & & \\
\hline Single living without children & $1155(66.8)$ & $917(56.7)$ & $222(54.8)$ & $458(58.6)$ & $588(68.9)$ & & & \\
\hline Single $e^{e}$ living with children & $148(8.6)$ & $236(14.6)$ & $80(19.8)$ & $133(17.0)$ & $127(14.9)$ & & & \\
\hline Unemployment & & & & & & & & \\
\hline No & $1177(68.1)$ & $1080(66.8)$ & $323(79.8)$ & $576(73.8)$ & $813(95.2)$ & $288.3(<0.001)$ & $248.5(<0.001)$ & 0.029 \\
\hline $1-180$ days & $419(24.2)$ & $425(26.3)$ & $72(17.8)$ & $170(21.8)$ & $36(4.2)$ & & & \\
\hline$>180$ days & $133(7.7)$ & $111(6.9)$ & $10(2.5)$ & $35(4.5)$ & $<10(0.6)$ & & & \\
\hline Clinical characteristics & & & & & & & & \\
\hline Somatic inpatient care ${ }^{f, g}$ & & & & & & & & \\
\hline No & $1160(67.1)$ & $862(53.3)$ & $151(37.3)$ & $349(44.7)$ & $298(34.9)$ & $310.8(<0.001)$ & $99.6(<0.001)$ & 0.012 \\
\hline Yes & $569(32.9)$ & $754(46.7)$ & $254(62.7)$ & $432(55.3)$ & $556(65.1)$ & & & \\
\hline Specialised somatic outpatient care & & & & & & & & \\
\hline No & $654(37.8)$ & $358(22.2)$ & $53(13.1)$ & $184(23.6)$ & $108(12.6)$ & $258.2(<0.001)$ & $49.2(<0.001)$ & 0.006 \\
\hline Yes & $1075(62.2)$ & $1258(77.8)$ & $352(86.9)$ & $596(76.4)$ & $746(87.4)$ & & & \\
\hline Mental diagnosis from in- or specia & ed outpatien & t care $\mathrm{f}^{\mathrm{f} g}$ & & & & & & \\
\hline No & 1049 (60.7) & $826(51.1)$ & $106(26.2)$ & 235 (30.1) & 153 (17.9) & $1068.8(<0.001)$ & $435.0(<0.001)$ & 0.052 \\
\hline
\end{tabular}


Table 2 Distributions and associations of socio-demographic and clinical characteristics in each trajectory group of annual months of sickness absence and disability pension (SA/DP) in individuals with suicide attempt treated in inpatient care in 2007-2009 in Sweden $(N=5385)$ (Continued)

\begin{tabular}{|c|c|c|c|c|c|c|c|c|}
\hline \multirow[t]{3}{*}{ Characteristics } & $\begin{array}{l}\text { Lowest } \\
\text { constant }\end{array}$ & $\begin{array}{l}\text { Intermediate } \\
\text { constant }\end{array}$ & $\begin{array}{l}\text { High } \\
\text { decreasing }\end{array}$ & $\begin{array}{l}\text { Low } \\
\text { increasing }\end{array}$ & $\begin{array}{l}\text { High } \\
\text { constant }\end{array}$ & \multirow[t]{3}{*}{$\begin{array}{l}\text { Pearson's Chi-Square } \\
(p \text {-value })^{k}\end{array}$} & \multirow[t]{3}{*}{$\begin{array}{l}\text { Log-likelihood test } \\
\text { Chi-Square } \\
(p \text {-value })^{\mid}\end{array}$} & \multirow[t]{3}{*}{$\begin{array}{l}\text { Diff. in } \\
\mathrm{R}^{2 *}\end{array}$} \\
\hline & n (\%) & n (\%) & n (\%) & n (\%) & n (\%) & & & \\
\hline & $1729(32.1)$ & $1616(30.0)$ & $405(7.5)$ & $781(14.5)$ & $854(15.9)$ & & & \\
\hline $\begin{array}{l}\text { Schizophrenia non-affective } \\
\text { psychoses }\end{array}$ & $18(1.0)$ & $10(0.6)$ & $<10(1.7)$ & $32(4.1)$ & $85(10.0)$ & & & \\
\hline Affective disorders & $122(7.1)$ & $245(15.2)$ & $88(21.7)$ & $198(25.4)$ & $148(17.3)$ & & & \\
\hline Substance abuse & $340(19.7)$ & $255(15.8)$ & $85(21.0)$ & $107(13.7)$ & $126(14.8)$ & & & \\
\hline $\begin{array}{l}\text { Neurotic, somatoform } \\
\text { and stress-related disorders }\end{array}$ & $143(8.3)$ & $219(13.6)$ & $81(20.0)$ & $130(16.6)$ & $160(18.7)$ & & & \\
\hline Personality disorders & $15(0.9)$ & $28(1.7)$ & $26(6.4)$ & $47(6.0)$ & $97(11.4)$ & & & \\
\hline Other mental disorders & $42(2.4)$ & $33(2.0)$ & $12(3.0)$ & $32(4.1)$ & $85(10.0)$ & & & \\
\hline \multicolumn{9}{|l|}{ Antidepressants ${ }^{\mathrm{h}, \mathrm{i}}$} \\
\hline No antidepressants & $1315(76.1)$ & $906(56.1)$ & $137(33.8)$ & $268(34.3)$ & $267(31.3)$ & $811.6(<0.001)$ & $93.6(<0.001)$ & 0.011 \\
\hline Low doses & $170(9.8)$ & $232(14.4)$ & $73(18.0)$ & $148(19.0)$ & 109 (12.8) & & & \\
\hline Moderate doses & $159(9.2)$ & $306(18.9)$ & $114(28.1)$ & $208(26.6)$ & $233(27.3)$ & & & \\
\hline High doses & $85(4.9)$ & $172(10.6)$ & $81(20.0)$ & $157(20.1)$ & $245(28.7)$ & & & \\
\hline \multicolumn{9}{|l|}{ Anxiolytics ${ }^{h, i}$} \\
\hline No Anxiolytics & $1443(83.5)$ & $1185(73.3)$ & $212(52.3)$ & $436(55.8)$ & $374(43.8)$ & $732.7(<0.001)$ & $47.6(<0.001)$ & 0.006 \\
\hline Low doses & $241(13.9)$ & $373(23.1)$ & $128(31.6)$ & $273(35.0)$ & $273(32.0)$ & & & \\
\hline Moderate doses & $28(1.6)$ & $36(2.2)$ & $41(10.1)$ & $50(6.4)$ & $112(13.1)$ & & & \\
\hline High doses & $17(1.0)$ & $22(1.4)$ & $24(5.9)$ & $22(2.8)$ & $95(11.1)$ & & & \\
\hline \multicolumn{9}{|l|}{ Sedatives $^{\mathrm{h}, \mathrm{i}}$} \\
\hline No sedatives & $1388(80.3)$ & $1118(69.2)$ & $184(45.4)$ & $369(47.2)$ & $305(35.7)$ & $942.1(<0.001)$ & $113.7(<0.001)$ & 0.013 \\
\hline Low doses & $242(14.0)$ & $339(21.0)$ & $103(25.4)$ & $224(28.7)$ & $172(20.1)$ & & & \\
\hline Moderate doses & $70(4.0)$ & $113(7.0)$ & $63(15.6)$ & $119(15.2)$ & $190(22.2)$ & & & \\
\hline High doses & $29(1.7)$ & $46(2.8)$ & 55 (13.6) & $69(8.8)$ & $187(21.9)$ & & & \\
\hline \multicolumn{9}{|l|}{ Method of suicide attempt ${ }^{g, j}$} \\
\hline Violent suicide attempt & $272(15.7)$ & $190(11.8)$ & $32(7.9)$ & $78(10.0)$ & 99 (11.6) & $30.4(<0.001)$ & $5.3(0.26)$ & 0.001 \\
\hline Non-violent suicide attempt & $1457(84.3)$ & $1426(88.2)$ & $373(92.1)$ & $703(90.0)$ & $755(88.4)$ & & & \\
\hline
\end{tabular}

* Difference in Nagelkerke pseudo $\mathrm{R}^{2}$ between model including tested variable and model without tested variable. Nagelkerke pseudo $\mathrm{R}^{2}$ for full model is 0.436

${ }^{a}$ Measured on 31-Dec of the year preceding suicide attempt

b Including other Northern European countries than Sweden and the rest of EU25

'Type of living area: big cities (Stockholm, Gothenburg and Malmö); medium sized cities (cities with more than 90,000 inhabitants within 30 km

distance from the centre of the city); small cities/villages/rural

d Married includes all living with partner; cohabiting

e Single comprises divorced, separated, and widowed

${ }^{f}$ Measured four years before suicide attempt

g See methods section for ICD-10 codes

$\mathrm{h}$ Measured during the year preceding the suicide attempt

i See methods section for ATC codes

${ }^{j}$ Measured at index suicide attempt

${ }^{\mathrm{k}}$ Crude model

I Mutually adjusted model

socio-demographic (sex, age, education, country of birth, type of living area, family situation, and unemployment) and clinical factors (in- or specialised outpatient somatic and mental care and psychiatric medication), except for method of suicide attempt, were significantly associated with different trajectory groups $\left(R^{2}=0.436, P<0.05\right)$ among suicide attempters in the adjusted analyses. Young suicide attempters with low levels of SA/DP tended to be unemployed with better level of education, whereas a larger proportion of those with high levels of 
SA/DP had schizophrenia/non-affected psychoses or personality disorders and lower levels of education.

\section{Methodological considerations}

To the best of our knowledge, this is the first study to investigate different trajectories of SA/DP before and after suicide attempt, compared with individuals without suicide attempt. The population-based cohort design, including all young adults aged $25-40$ years in the entire country of Sweden, offered satisfactory statistical power for the analyses of trajectories of SA/DP months. Another strength was the use of high quality nationwide register data on suicide attempt, SA/DP and a wide range of socio-demographic and clinical factors [20, 21]. The use of register data also minimised the risk of recall bias regarding exposure and outcome.

Some limitations of the study are important to mention. Information on sick-leave spells $<14$ days among employed individuals was not available. This means that for employed individuals the number of SA days contributing to the combined number of SA/DP days might be an underestimation. Only suicide attempts that required inpatient care were included, which only covered the medically more serious cases. This means that suicide attempters who were treated at other healthcare facilities or did not seek healthcare were not included.

\section{Trajectories of SA/DP}

Our findings showed considerable heterogeneity with regard to patterns of SA/DP before and after suicide attempt. In the suicide attempt cohort, we found that two thirds of the individuals had either no SA/DP or few months of SA/DP over time while one group had constantly high SA/DP levels (16\%). The absolute majority of individuals without suicide attempt (94\%) had no or very low levels of SA/DP during the 8 years of observation.

These findings are comparable to results from a recently published study, which showed five different trajectories of SA/DP before suicide [10]. However, a larger proportion of individuals $(30 \%)$ in that previous study had stable high levels of SA/DP months over time before suicide, which was twice as much as among suicide attempters in the current study. Also, this group of individuals had around 11 months of SA/DP during 4 years before suicide [10], which was moderately higher than the corresponding group in our findings. On the other hand, there was a larger proportion of young suicide attempters with constantly no or low level of SA/ DP months (62\%) in our study compared to the low SA/ DP trajectory (46\%) before suicide [10]. In the current study, only young adults aged between 25 and 40 at the time of suicide attempt were included. As a result of the peculiarities of the social insurance system in Sweden, younger individuals might be less eligible to SA due to a lack of labour market attachment [13, 22]. That could explain why high SA/DP was less frequent in this study compared to the study on suicide completers. Other explanations might be differences in the morbidity level between suicide attempters and suicide completers [2, 23, 24].

Our results further indicate that SA/DP trajectories can be characterised by different socio-demographic and clinical factors. The specific mental diagnosis before suicide attempt showed the strongest associations in the full model. Mental disorders are very common diagnoses behind SA/DP [13]. Moreover, mental disorders represent the most important risk factors for suicidal behaviour $[2,23]$. Therefore, it is reasonable to find that a larger proportion of individuals in the "High constant" group had psychiatric health care before their suicide attempt, particularly due to schizophrenia, non-affective psychoses or personality disorders and high doses of antidepressants, anxiolytics, and sedatives. The "High decreasing" group with relatively high levels of SA/DP months before suicide attempt also had low/moderate doses of psychiatric medication. This might reflect a higher morbidity level and lower levels of work capacity requiring more SA/DP days and increasing the risk of suicide attempt. In addition, the majority of individuals in these groups had previous specialised somatic healthcare. This is in line with studies on somatic comorbidity in suicide attempters [2].

On the other hand, the "Lowest constant" group consisted of individuals with fewer previous somatic and mental healthcare and less use of psychiatric medication in comparison with other groups. It might be due to the fact that the group also include more younger individuals (below 30 years of age) and more men. Young people may have better health conditions than older ones and thus use less healthcare and medication, however, young men might also be less likely to seek help and disclose health problems compared to their female counterparts $[25,26]$. Therefore, undetected mental or somatic disorders are likely in this group. Furthermore, a larger proportion of individuals in this group was born in a non-European country. It might be possible that they were less eligible to SA due to their lower level of labour market attachment or had less access to healthcare than individuals from the host country [27]. On the other hand, a larger proportion of unemployed individuals were found in the "Lowest constant" and the "Intermediate constant" group compared to other groups. This might indicate that young suicide attempters were marginalised at labour market even though the levels of SA/DP were low. This result is in line with previous research which has demonstrated an association between unemployment and suicide attempt on the one hand and with SA/DP on the other hand $[2,28]$.

Interestingly, two fluctuating trajectory groups, "High decreasing" and "Low increasing" were found in this study. 
The "Low increasing" group included more individuals with a diagnosis of affective disorders and low doses of psychiatric medication use, which might be associated with subsequent increasing SA/DP months and suicide attempt. On the other hand, it is surprising that about $8 \%$ of individuals had increasing SA/DP months in the beginning of the observation period and their SA/DP months declined from 2 years before suicide attempt. A considerable proportion of this group of individuals had previous in- or specialised outpatient somatic health care, substance abuse, and prescribed psychiatric medication. The decreasing SA/DP months might reflect improvement in health conditions, decreased help-seeking or inadequate treatment or rehabilitation. Suicide attempt as an impulsive behaviour could be also associated with other factors than healthcare characteristics [2]. Thus, further studies are required to gain more knowledge in this trajectory group.

\section{Conclusions}

This study showed five different trajectories of SA/DP over a period of 8 years before and after suicide attempt among young adults. While two thirds of the suicide attempters had no or low levels of SA/DP over time, 15\% had constantly high levels. In contrast, the absolute majority (94\%) of young adults without suicide attempt showed no or low levels of SA/DP during the observation time. Sociodemographic and clinical factors were associated with different SA/DP trajectories, especially earlier diagnosed schizophrenia/non-affective psychoses, personality disorders, and unemployment. It seems that marginalisation at the labour market is considerable for suicide attempters even though the levels of SA/DP are low.

\section{Abbreviations \\ ATC: Anatomical therapeutic chemical; BIC: Bayesian information criterion; DDD: Defined daily dose; DP: Disability pension; ICD: International classification of diseases; LISA: Longitudinal integration database for health insurance and labour market studies; SA: Sickness absence}

\section{Acknowledgements}

Not applicable

\section{Funding}

This study was funded by the Swedish Research Council with dnr 521-2014-3335. The funders had no role in study design, data collection, analysis, decision to publish, or preparation of the manuscript.

\section{Availability of data and materials}

The data cannot be made publically available. According to the Swedish Ethical Review Act, the Personal Data Act, and the Administrative Procedure Act, data can only be made available, after legal review, for researchers who meet the criteria for access to this type of sensitive and confidential data. Readers may contact professor Kristina Alexanderson

(kristina.alexanderson@ki.se) regarding questions about this.

\section{Authors' contributions}

MW, MH, SR, TN and EMR contributed to the design of the study and the statistical analysis plan. MW was responsible for data management and data analysis. MW and EMR drafted the manuscript and all authors were involved in interpretation of the data, critical revision, and approval of the final content before submission.

\section{Ethics approval and consent to participate}

The study population was based on linkage of several public national registers. Ethical vetting is always required when using register data in Sweden. The ethical vetting is performed by the Regional Ethical Review Board of Karolinska Institutet and the risk appraisal associated with the Law on Public Disclosure and Secrecy is done by data owners. The Regional Ethical Review Board of Karolinska Institutet, Stockholm, Sweden can however waive the requirement to consult the study subjects (or in case of minors/children the next of kin, carers or guardians) directly to obtain their informed consent, and will often do so if the research is supported by the ethical review board and the data has already been collected in some other context. According to these standards in Sweden this project has been evaluated and approved by the Regional Ethical Review Board of Karolinska Institutet, Stockholm, Sweden (review number 2007/762-31).

\section{Consent for publication}

Not applicable

\section{Competing interests}

The authors declare that they have no competing interests.

\section{Publisher's Note}

Springer Nature remains neutral with regard to jurisdictional claims in published maps and institutional affiliations.

\section{Author details}

'Division of Insurance Medicine, Department of Clinical Neuroscience, Karolinska Institutet, SE 17177 Stockholm, Sweden. ${ }^{2}$ Center for Public Health, Department of Social and Preventive Medicine, Medical University Vienna, Vienna, Austria.

Received: 7 June 2017 Accepted: 30 November 2017

Published online: 08 December 2017

\section{References}

1. WHO. Preventing suicide. A global imperative: World Health Organization. 2005 http://apps.who.int/iris/bitstream/10665/131056/1/9789241564779_ eng.pdf?ua=1\&ua=1. Accessed 14 Jun 2017.

2. Skegg K. Self-harm. Lancet. 2005 Oct 22-28;366(9495):1471-83.

3. Landstedt $\mathrm{E}$, Gillander GK. Deliberate self-harm and associated factors in 17year-old Swedish students. Scand J Public Health. 2011;39(1):17-25.

4. Niederkrotenthaler $\mathrm{T}$, Tinghog $\mathrm{P}$, Alexanderson $\mathrm{K}$, et al. Future risk of labour market marginalization in young suicide attempters-a population-based prospective cohort study. Int J Epidemiol. 2014;43(5):1520-30.

5. Niederkrotenthaler T, Tinghog P, Goldman-Mellor S, et al. Medical and social determinants of subsequent labour market marginalization in young hospitalized suicide attempters. PLoS One. 2016;11(1):e0146130.

6. Bjorkenstam C, Alexanderson K, Bjorkenstam E, et al. Diagnosis-specific disability pension and risk of all-cause and cause-specific mortality-a cohort study of 4.9 million inhabitants in Sweden. BMC Public Health. 2014;14:1247.

7. Wang M, Alexanderson $\mathrm{K}$, Runeson B, et al. Are all-cause and diagnosisspecific sickness absence, and sick-leave duration risk indicators for suicidal behaviour? A nationwide register-based cohort study of 4.9 million inhabitants of Sweden. Occup Environ Med. 2014;71(1):12-20.

8. Wang M, Alexanderson K, Runeson B, et al. Sick-leave measures, sociodemographic factors and health care as risk indicators for suicidal behavior in patients with depressive disorders - a nationwide prospective cohort study in Sweden. J Affect Disord. 2015;173:201-10.

9. Wang M, Alexanderson K, Runeson B, et al. Morbidity and suicide mortality following sick leave in relation to changes of social insurance regulations in Sweden. Eur J Pub Health. 2016;26(6):1061-9.

10. Wang M, Bjorkenstam C, Alexanderson K, et al. Trajectories of work-related functional impairment prior to suicide. PLoS One. 2015;10(10):e0139937.

11. Mittendorfer-Rutz E, Rasmussen F, Wasserman D. Restricted fetal growth and adverse maternal psychosocial and socioeconomic conditions as risk factors for suicidal behaviour of offspring: a cohort study. Lancet. 2004 Sep 25-Oct 1;364(9440):1135-40.

12. Chen $\mathrm{Q}$, Sjolander A, Runeson B, et al. Drug treatment for attention-deficit/ hyperactivity disorder and suicidal behaviour: register based study. BMJ. 2014;348:93769.

13. The Swedish Social Insurance Agency. Social Insurance in Figures 2016. 
14. WHO Collaborating Center for Drug Statistics Methodology. Definition and general consideration.: WHOCC; 2017; https://www.whocc.no/ddd/ definition_and_general_considera/. Accessed 10 Oct 2016.

15. Torniainen M, Mittendorfer-Rutz E, Tanskanen A, et al. Antipsychotic treatment and mortality in schizophrenia. Schizophr Bull. 2015;41(3):656-63.

16. Mittendorfer-Rutz E, Wasserman D, Rasmussen F. Fetal and childhood growth and the risk of violent and non-violent suicide attempts: a cohort study of 318,953 men. J Epidemiol Community Health. 2008;62(2):168-73.

17. WHO. The ICD-10 classification of mental and Behavioural disorders. 2013. http://www.who.int/classifications/icd/en/bluebook.pdf. Accessed 1 Nov 2016.

18. Jones B, Nagin D, Roeder K. A SAS procedure based on mixture models for estimating developmental trajectories. Sociol Methods Res. 2001;29(3):374-93.

19. Cote S, Tremblay RE, Nagin D, et al. The development of impulsivity, fearfulness, and helpfulness during childhood: patterns of consistency and change in the trajectories of boys and girls. J Child Psychol Psychiatry. 2002; 43(5):609-18.

20. Ludvigsson JF, Andersson E, Ekbom A, et al. External review and validation of the Swedish national inpatient register. BMC Public Health. 2011;11:450.

21. The National Board of Health and Welfare. Dödsorsaksregistret (the cause of death register) (In Swedish). Stockholm, Sweden.2015. www.socialstyrelsen. se. Accessed 20 Sep 2016.

22. Karlsson N, Borg K, Carstensen J, et al. Risk of disability pension in relation to gender and age in a Swedish county; a 12-year population based, prospective cohort study. Work. 2006;27(2):173-9.

23. Hawton K, van Heeringen K. Suicide Lancet. 2009;373(9672):1372-81.

24. Hawton K, Saunders KE, O'Connor RC. Self-harm and suicide in adolescents. Lancet. 2012;379(9834):2373-82.

25. Burns BJ, Ryan Wagner H, Gaynes BN, et al. General medical and specialty mental health service use for major depression. Int J Psychiatry Med. 2000; 30(2):127-43.

26. Angst J, Gamma A, Gastpar M, et al. Gender differences in depression. Epidemiological findings from the European DEPRES I and II studies. Eur Arch Psychiatry Clin Neurosci. 2002;252(5):201-9.

27. Dunlavy AC, Rostila M. Health inequalities among workers with a foreign background in Sweden: do working conditions matter? Int J Environ Res Public Health. 2013;10(7):2871-87.

28. Helgesson M, Johansson B, Nordqvist T, et al. Sickness absence at a young age and later sickness absence, disability pension, death, unemployment and income in native Swedes and immigrants. Eur J Pub Health. 2015;25(4):688-92.

\section{Submit your next manuscript to BioMed Central and we will help you at every step:}

- We accept pre-submission inquiries

- Our selector tool helps you to find the most relevant journal

- We provide round the clock customer support

- Convenient online submission

- Thorough peer review

- Inclusion in PubMed and all major indexing services

- Maximum visibility for your research

Submit your manuscript at www.biomedcentral.com/submit

) Biomed Central 